\title{
Directly imaging steeply-dipping fault zones in geothermal fields with multicomponent seismic data
}

\author{
Ting Chen, Lianjie Huang \\ Geophysics Group, Los Alamos National Laboratory, Los Alamos, NM 87545, USA
}

\begin{abstract}
For characterizing geothermal systems, it is important to have clear images of steeply-dipping fault zones because they may confine the boundaries of geothermal reservoirs and influence hydrothermal flow. Elastic reverse-time migration (ERTM) is the most promising tool for subsurface imaging with multicomponent seismic data. However, conventional ERTM usually generates significant artifacts caused by the cross correlation of undesired wavefields and the polarity reversal of shear waves. In addition, it is difficult for conventional ERTM to directly image steeply-dipping fault zones. We develop a new ERTM imaging method in this paper to reduce these artifacts and directly image steeply-dipping fault zones. In our new ERTM method, forward-propagated source wavefields and backwardpropagated receiver wavefields are decomposed into compressional $(\mathrm{P})$ and shear (S) components. Each component of these wavefields is separated into left- and right-going, or downgoing and upgoing waves. The cross correlation imaging
\end{abstract}

\footnotetext{
${ }^{*}$ Corresponding author: Ting Chen email: tchen@lanl.gov phone 1-505-667-8938, fax 1-505-667-3494

Los Alamos National Laboratory, MS D446, Los Alamos, NM 87545, USA

Email addresses: tchen@lanl.gov (Ting Chen), ljh@lanl.gov (Lianjie Huang)
} 
condition is applied to the separated wavefields along opposite propagation directions. For converted waves (P-to-S or S-to-P), the polarity correction is applied to the separated wavefields based on the analysis of Poynting vectors. Numerical imaging examples of synthetic seismic data demonstrate that our new ERTM method produces high-resolution images of steeply-dipping fault zones.

Keywords:

Steeply-dipping fault, seismic imaging, elastic reverse-time migration, wavefield separation, geothermal exploration, Poynting vector 


\begin{tabular}{|c|c|}
\hline \multicolumn{2}{|c|}{ Nomenclature } \\
\hline ERTM & elastic reverse-time migration \\
\hline $\mathrm{P}$ & compressional wave \\
\hline $\mathrm{S}$ & shear wave \\
\hline $\mathbf{F}$ & Poynting vector \\
\hline $\mathbf{u}$ & wavefield displacement or particle velocity \\
\hline$p$ & wavefield quantity \\
\hline$s(\mathbf{x}, \tau)$ & forward propagated source wavefield \\
\hline$r(\mathbf{x}, t-\tau)$ & backward propagated receiver wavefield \\
\hline$I$ & migration image \\
\hline$I^{\mathrm{d}}$ & downward-looking migration image \\
\hline$I^{\mathrm{u}}$ & upward-looking migration image \\
\hline$I^{1}$ & left-looking migration image \\
\hline$I^{\mathrm{r}}$ & right-looking migration image \\
\hline$I_{\mathrm{PP}}$ & $\begin{array}{l}\text { migration image constructed using the compressional source } \\
\text { wavefield and the compressional receiver wavefield }\end{array}$ \\
\hline$I_{\mathrm{PS}}$ & $\begin{array}{l}\text { migration image constructed using the compressional source } \\
\text { wavefield and the shear receiver wavefield }\end{array}$ \\
\hline$I_{\mathrm{SP}}$ & $\begin{array}{l}\text { migration image constructed using the shear source wavefield } \\
\text { and the compressional receiver wavefield }\end{array}$ \\
\hline
\end{tabular}


$I_{\mathrm{SS}} \quad$ migration image constructed using the shear source wavefield and the shear receiver wavefield 


\section{Introduction}

Faults play an important role in geothermal fields. High-permeability faults provide preferential pathways for geothermal fluids, and are the primary control for efficient fluid flow and heat transfer (e.g., Goyal and Kassoy, 1980; López and Smith, 1995; Corbel et al., 2012). Faults may also act as barriers to fluid flow by juxtaposing impermeable lithologies against the reservoir rock (e.g., Knipe, 1992, Barnicoat et al. 2009). The existence of faults has a significant influence on the patterns of convection within the geothermal reservoir (e.g., Caine and Forster, 1999: Corbel et al. 2012). Faults can also produce earthquakes when the stress state in the field changes, causing damage to property and raising concerns of local communities (e.g., Megies and Wassermann, 2014; Wassing et al., 2014). Therefore, knowing the fault locations is crucial for understanding the hydrothermal flow in the geothermal system, conducting risk assessments, and planning geothermal operations.

Different techniques are available for detecting faults at depth in geothermal fields, including geological, gravity, magnetic, electromagnetic, and seismic surveys (Hatherton et al., 1966). Seismic methods are excellent tools for detecting faults because it could effectively map the locations and extension of buried faults both laterally and vertically.

As a standard data processing technique for seismic reflection data, conventional seismic migration methods, such as Kirchhoff migration, use primary reflection signals from subsurface interfaces to obtain images of impedance contrasts within geological structures. These conventional seismic migration methods can directly image horizontal layers and shallow-dipping faults. For steeply-dipping faults, as is usually found in geothermal fields (e.g., Huenges, 2010), none or only 
a very small portion of the primary reflection signals from these faults are recorded in surface seismic reflection data. Multiple reflections that illuminate these faults are much weaker than the primary reflections from stratigraphic layers. Therefore, it is a challenge to directly image steeply-dipping faults. The locations of steeply-dipping faults are, instead, usually inferred based on the offset of imaged stratigraphic layers. In many cases, because of the ambiguity in the offset of the stratigraphic layers, these inferences are subjective and inaccurate. Therefore, it is important and highly desirable to develop a seismic imaging method that is capable of directly imaging steeply-dipping fault zones in geothermal fields.

Reverse-time migration (RTM) is the most promising tool for high-resolution images of complex subsurface structures including steeply-dipping faults (e.g., Baysal et al., 1983; McMechan, 1983; Whitmore, 1983; Tan and Huang, 2014). RTM solves the full scalar-wave equation in heterogeneous media for forward propagation of source wavefields and backward propagation of recorded seismic reflection data from receivers. Thus it can handle complex wave phenomena including multiple reflections, and has no dip limitation. RTM is commonly employed for single-component seismic data. Multicomponent seismic surveys have developed rapidly during recent years (Gaiser et al., 2001). Multicomponent seismic data contain more complete information about seismic wavefields, and are useful in many applications that are not achievable with single-component data (Stewart et al., 2002, 2003). Performing elastic reverse-time migration (ERTM) rather than RTM is necessary to take full advantage of the additional information contained in multicomponent seismic data. ERTM solves the elastic-wave equation in heterogeneous media, and can properly handle the conversions between compressional (P) and shear (S) waves (Chang and McMechan, 1987). ERTM 
has been shown to provide better images of subsurface structures than conventional seismic migration and RTM (e.g., Lu et al., 2009; Huang and Albrecht, 2011; Huang et al., 2011).

a Despite powerful imaging capabilities, ERTM faces some challenges (Yoon et al. 2004) for high-resolution imaging of complex subsurface structures. One of the challenges is the appearance of low-wavenumber artifacts in migration images (P-to-P or S-to-S) with the conventional full-wavefield zero-lag cross-correlation imaging condition (Claerbout, 1985). These artifacts are caused by the cross correlation of diving waves and their corresponding backscattered waves at locations other than the reflectors. Such artifacts are particularly strong in shallow layers or regions with large impedance contrasts, contaminating images of subsurface structures. Different approaches have been developed to suppress these lowwavenumber image artifacts. Filtering applied after imaging using filters such as bandpass, derivative, Laplacian, and more advanced least-square filters can reduce the low-wavenumber artifacts (Youn and Zhou, 2001; Mulder and Plessix, 2004; Guitton et al., 2007). However, the filtering may change the character of reflections such as phase and spectrum, increase high-wavenumber artifacts, or remove useful information. Reducing the reflections by smoothing the velocity model (Loewenthal et al., 1987) or using a nonreflecting wave equation (Baysal et al., 1984) can also suppress the artifacts, but introduce unrealistic wavefields. Modifying imaging conditions is another method to attenuate or remove the artifacts. Such methods include normalizing the conventional imaging condition by source or receiver illuminations (Kaelin et al., 2007), and separating or weighting wavefields traveling in different directions (e.g., Yoon et al., 2004; Costa et al., 2009; Liu et al., 2011). 
Another challenge for ERTM is the polarity reversal problem for images obtained with converted waves (P-to-S or S-to-P). Because the polarity directions of $\mathrm{S}$ waves change across the normal incidence direction, when we stack migration images from different common-shot seismic data, mixed signs of polarity lead to destructive images. To obtain coherent images for the converted waves, we need to apply a polarity correction. Because the signs of polarity depend on the direction of the incident waves relative to a reflector, it is straightforward to correct for the polarity reversal by analyzing the propagation directions of the waves. Two popular methods can be used for this purpose. One applies the extended imaging condition to extract the angle information (Rickett and Sava, 2002; Sava and Fomel, 2006; Yan and Sava, 2008; Fomel, 2011), and the other uses Poynting vectors to obtain wave propagation directions (Dickens and Winbow, 2011; Vyas et al. 2011; Yoon et al., 2011; Du et al., 2012). The extended imaging condition method with the ability to handle multipathing is generally more accurate in complex geology, but it has lower angular resolution and is computationally expensive. The Poynting-vector method has high angular resolution and is computationally efficient, but cannot handle multipathing and is difficult to accurately estimate propagation directions for complicated wavefields (Patrikeeva and Sava, 2013).

We develop a new imaging condition for 2D ERTM by combining wavefield separation with the Poynting vector method. We separate the forward-propagated source wavefields and backward-propagated receiver wavefields along the vertical direction to obtain the downgoing and upgoing waves, and along the horizontal direction to obtain the left-going and right-going waves. ERTM with our new imaging condition can directly image steeply-dipping fault zones using multicompo- 
119

nent seismic data, while eliminating the low-wavenumber artifacts and correcting the polarity reversal efficiently and more accurately. We apply our method to synthetic elastic seismic reflection data for a geophysical model from the Soda Lake geothermal site containing several steeply-dipping fault zones, and demonstrate the capability of our new method for directly imaging steeply-dipping fault zones.

\section{Method}

\subsection{Elastic reverse-time migration}

The first step of ERTM is elastic wavefield extrapolation. We compute elastic wavefields in an isotropic, non-attenuative medium using a staggered-grid highorder finite-difference scheme in the time domain. A perfectly matched layer (PML) approach is implemented as an absorbing boundary condition. The source wavefield is propagated forward in time, and the receiver wavefield is extrapolated backward in time using the recorded multicomponent seismic data as boundary values.

After the wavefield extrapolation (forward or backward propagation), we decompose both the source and receiver wavefields into compressional $(\mathrm{P})$ and shear (S) components based on Helmholtz decomposition (Dellinger and Etgen, 1990):

$$
P=\nabla \cdot \mathbf{u}
$$

$$
\mathbf{S}=\nabla \times \mathbf{u}
$$

where $\mathbf{u}$ is the extrapolated elastic wavefield. It can be the vector field of either displacement or particle velocity in practice. The scalar $P$ represents the curlfree part of the wavefield. The vector $\mathbf{S}$ describes the divergence-free part of the 
wavefield. In two dimensions, the vector $\mathbf{S}$ has only one non-zero component and can also be treated as a scalar.

The conventional imaging condition in ERTM is implemented as

$$
I(\mathbf{x})=\int_{0}^{t} s(\mathbf{x}, \tau) r(\mathbf{x}, t-\tau) d \tau,
$$

where $s(\mathbf{x}, \tau)$ is the forward propagated source wavefield at location $\mathbf{x}$ and time $\tau$, $r(\mathbf{x}, t-\tau)$ denotes the backward propagated receiver wavefield, and $t$ represents the maximum record time in seismic traces. By cross correlating different wave modes (P or $\mathrm{S}$ ) of the source and receiver wavefields, we can obtain four different images: compressional-to-compressional $\left(I_{\mathrm{PP}}\right)$, compressional-to-shear $\left(I_{\mathrm{PS}}\right)$, shear-to-compressional $\left(I_{\mathrm{SP}}\right)$, and shear-to-shear $\left(I_{\mathrm{SS}}\right)$ images. Comparing with simple cross correlation of Cartesian components of the wavefields, the images formed with cross correlation of decomposed $\mathrm{P}$ and $\mathrm{S}$ components are free of artifacts caused by the crosstalk between different wave modes, and are easier to interpret in a physical sense (Yan and Sava, 2008).

\subsection{Wavefield separation}

If we use the conventional imaging condition in Eq. (3), the resulting images $I_{\mathrm{PP}}$ and $I_{\mathrm{SS}}$ will have large low-wavenumber artifacts. These artifacts are caused by the undesired cross correlation between diving waves and backscattered waves.

To remove these low-wavenumber artifacts, we can modify the imaging conditions with separated wavefields (Denli and Huang, 2008; Liu et al., 2011). After wave-mode decomposition, we use a frequency-wavenumber (f-k) filter to separate $\mathrm{P}$ and $\mathrm{S}$ components from both the source and receiver wavefields along horizontal and vertical directions, and then use the separated downgoing, upgoing, 
left-going, and right-going wavefields to form multiple images. The downwardlooking $\left(I^{\mathrm{d}}\right)$, upward-looking $\left(I^{\mathrm{u}}\right)$, left-looking $\left(I^{\mathrm{l}}\right)$, and right-looking $\left(I^{\mathrm{r}}\right)$ images are constructed using the cross correlations of the separated wavefields propagating in the opposite directions:

$$
\begin{aligned}
I^{\mathrm{d}}(\mathbf{x}) & =\int_{0}^{t} s^{+z}(\mathbf{x}, \tau) r^{-z}(\mathbf{x}, t-\tau) d \tau, \\
I^{\mathrm{u}}(\mathbf{x}) & =\int_{0}^{t} s^{-z}(\mathbf{x}, \tau) r^{+z}(\mathbf{x}, t-\tau) d \tau, \\
I^{\mathrm{l}}(\mathbf{x}) & =\int_{0}^{t} s^{-x}(\mathbf{x}, \tau) r^{+x}(\mathbf{x}, t-\tau) d \tau, \\
I^{\mathrm{r}}(\mathbf{x}) & =\int_{0}^{t} s^{+x}(\mathbf{x}, \tau) r^{-x}(\mathbf{x}, t-\tau) d \tau,
\end{aligned}
$$

where " $+z$ ", “ $-z$ ", “- $-x$ ", and " $+x$ ” designate downgoing, upgoing, left-going, and right-going wavefields, respectively. The wavefields $s$ and $r$ can represent either the $\mathrm{P}$ or $\mathrm{S}$ component, forming $\mathrm{PP}\left(I_{\mathrm{PP}}\right)$, PS $\left(I_{\mathrm{PS}}\right), \mathrm{SP}\left(I_{\mathrm{SP}}\right)$, and $\mathrm{SS}\left(I_{\mathrm{SS}}\right)$ images. $I^{\mathrm{d}}$ and $I^{\mathrm{u}}$ provide images of horizontal or shallow-dipping reflectors, while $I^{1}$ and $I^{\mathrm{r}}$ show vertical or steeply-dipping reflectors, such as steep faults. For surface seismic surveys, seismic sources are located at the Earth's surface, or above the subsurface structures, and the most useful source wave signals are downgoing instead of upgoing, so we use the combination of $I^{\mathrm{d}}, I^{\mathrm{l}}$, and $I^{\mathrm{r}}$ to form the complete images.

Since diving waves and time-reversed backscattered waves have the same propagation directions, using only the separated waves along opposite directions instead of the full wavefields to form migration images, we can eliminate the undesired cross correlation between source and receiver wavefields and remove the low-wavenumber artifacts. The separation of wavefields along both horizontal and vertical directions and the imaging condition with only waves along oppo- 
site propagation directions also enable us to directly image steep faults, because steeply-dipping reflectors are illuminated mostly by weak multiple reflections, which are likely buried in other strong primary reflection signals without wavefield separation.

\subsection{Polarity correction}

Images $I_{\mathrm{PS}}$ and $I_{\mathrm{SP}}$, constructed using converted waves, do not contain lowwavenumber artifacts, because the diving waves and their corresponding backscattered waves are not in phase as a result of the different velocities of $\mathrm{P}$ and $\mathrm{S}$ waves. However, $I_{\mathrm{PS}}$ and $I_{\mathrm{SP}}$ may suffer from polarity issues.

Because of the polarity reversal of $\mathrm{S}$ waves, the S-wave component (Eq. (2)) has different directions on the opposite sides of the normal incidence point. Since we treat the S-wave component as a scalar in the imaging condition, the different directions of the S-wave component are shown with different signs in Eqs. (3) and (4). For $I_{\mathrm{SS}}$, that does not matter because both source $(s)$ and receiver $(r)$ wavefields change signs. For $I_{\mathrm{PS}}$ and $I_{\mathrm{SP}}$, the polarity reversal of $\mathrm{S}$ waves results in flipped signs on opposite sides of normal incidence points for each of the common-shot images. Because seismic incident waves from different shots have different normal incidence points, when we stack migration images from multiple common-shot gathers, the images become destructive rather than constructive or coherent.

To correct for the polarity reversal, we may first compute Poynting vectors for both source and receiver wavefields. The Poynting vector $\mathbf{F}$ describes the energy flux density, and can be calculated using

$$
\mathbf{F}=-\dot{p} \nabla p
$$


where $p$ is the wavefield quantity for $\mathrm{P}$ or $\mathrm{S}$ waves (Eqs. (1), (2)). The Poynting vectors calculated using Eq. (5) is more accurate than those obtained using the multiplication of stress and velocity (Du et al., 2012). The sign of the cross product between the Poynting vectors of the source and receiver wavefield $\mathbf{F}_{\mathrm{s}} \times \mathbf{F}_{\mathrm{r}}$ changes with the polarity distribution of $\mathrm{S}$ waves, and can be used to correct for polarity reversal as (Du et al., 2012)

$$
I(\mathbf{x})=\int_{0}^{t} s(\mathbf{x}, \tau) r(\mathbf{x}, t-\tau) \operatorname{sgn}\left(\mathbf{F}_{\mathrm{s}} \times \mathbf{F}_{\mathrm{r}}\right) d \tau .
$$

The Poynting vector analysis is a very attractive approach for correcting the polarity reversal because of its computational efficiency. This is crucial for largescale 3D migration imaging. However, it is difficult to accurately estimate Poynting vectors for complicated wavefields. In addition, because the Poynting vector assumes only one propagation direction at any time for a given point, it cannot handle multipathing. To alleviate these limitations, rather than directly using the imaging condition in Eq. (6), we estimate Poynting vectors for separated wavefields, and then apply the polarity correction to the separated wavefields to obtain downward-looking, upward-looking, left-looking, and right-looking images for $I_{\mathrm{PS}}$ and $I_{\mathrm{SP}}$ as:

$$
\begin{aligned}
& I^{\mathrm{d}}(\mathbf{x})=\int_{0}^{t} s^{+z}(\mathbf{x}, \tau) r^{-z}(\mathbf{x}, t-\tau) \operatorname{sgn}\left(\mathbf{F}_{\mathrm{s}}^{+z} \times \mathbf{F}_{\mathrm{r}}^{-z}\right) d \tau, \\
& I^{\mathrm{u}}(\mathbf{x})=\int_{0}^{t} s^{-z}(\mathbf{x}, \tau) r^{+z}(\mathbf{x}, t-\tau) \operatorname{sgn}\left(\mathbf{F}_{\mathrm{s}}^{-z} \times \mathbf{F}_{\mathrm{r}}^{+z}\right) d \tau, \\
& I^{\mathrm{l}}(\mathbf{x})=\int_{0}^{t} s^{-x}(\mathbf{x}, \tau) r^{+x}(\mathbf{x}, t-\tau) \operatorname{sgn}\left(\mathbf{F}_{\mathrm{s}}^{-x} \times \mathbf{F}_{\mathrm{r}}^{+x}\right) d \tau, \\
& I^{\mathrm{r}}(\mathbf{x})=\int_{0}^{t} s^{+x}(\mathbf{x}, \tau) r^{-x}(\mathbf{x}, t-\tau) \operatorname{sgn}\left(\mathbf{F}_{\mathrm{s}}^{+x} \times \mathbf{F}_{\mathrm{r}}^{-x}\right) d \tau .
\end{aligned}
$$

Because separated wavefields become simpler than the full wavefields, we are able to improve the accuracy of the computed Poynting vectors and enhance 
polarity-corrected images using Eq. (7). Computing Poynting vectors after wavefield separation can also help distinguish some multipathing with different propagating directions. Therefore, ERTM with the imaging condition shown in Eq. (7) is an efficient and effective method for directly imaging steep-dipping faults using multicomponent seismic data.

While we develop our new ERTM method for improving imaging of steeplydipping faults that are difficult for conventional migration to map, our new method can also image reflectors with any dip angles and faults with any thicknesses.

\section{Numerical examples}

Our focus is to directly image steeply-dipping fault zones in geothermal fields using multicomponent seismic data. First, we validate the effectiveness of our ERTM method for imaging faults using a simple 2D layered model with one vertical fault. Then we apply our method to image a 2D geophysical model for the Soda Lake geothermal site in Nevada.

\subsection{Vertical fault model}

We consider a four-layer model with an embedded vertical fault zone (Fig. 11). The middle two layers are displaced approximately $5 \mathrm{~m}$ vertically along the fault. The fault zone has a thickness of $20 \mathrm{~m}$. The P-wave velocity of the fault zone is $10 \%$ lower than that of the surrounding regions. S-wave velocities have similar structure to the P-wave velocities with a constant ratio of $V_{\mathrm{P}} / V_{\mathrm{S}}=1.73$. Such a fault with a dip angle as large as 90 degrees and small displacement is particularly difficult to image using conventional seismic migration methods that use only primary reflections for imaging. 


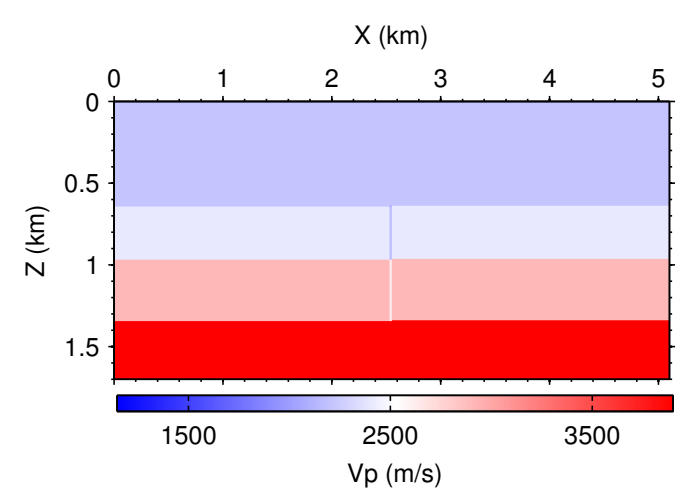

(a) P-wave velocity

We use a high-order finite-difference scheme to solve the elastic-wave equation to generate synthetic elastic reflection data for a surface seismic survey. A total of 34 explosive shots with an even spacing of $30 \mathrm{~m}$ are located on the top surface of the model. The source signal is a Ricker wavelet with a center frequency of $20 \mathrm{~Hz}$. The grid size of the model is $5 \mathrm{~m}$.

We apply our new ERTM method to the synthetic seismic data to perform migration imaging using the true velocity model. We then compare our migration images with those obtained using the conventional migration imaging condition. Fig. 2 shows a comparison of PP images within a region around the vertical fault zone. The image in Fig. 2a obtained using the conventional ERTM (Eq. (3)) is contaminated by high-amplitude background artifacts, which mask a large part of the structures. The artifacts overwhelm the images of reflectors so the image appears all blue with a symmetric red-white-blue colorbar.

If we separate the source and receiver wavefields into downgoing and upgoing components, and perform cross correlation only between the downgoing source

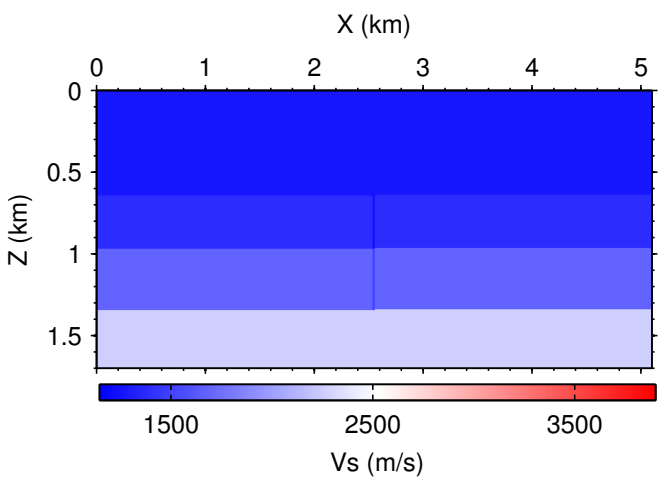

(b) S-wave velocity

Fig. 1. A model with a vertical fault. (a) P-wave velocity and (b) S-wave velocity. 


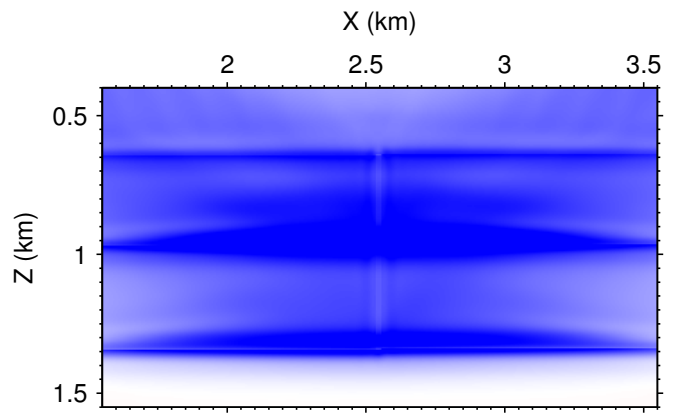

(a) Conventional ERTM

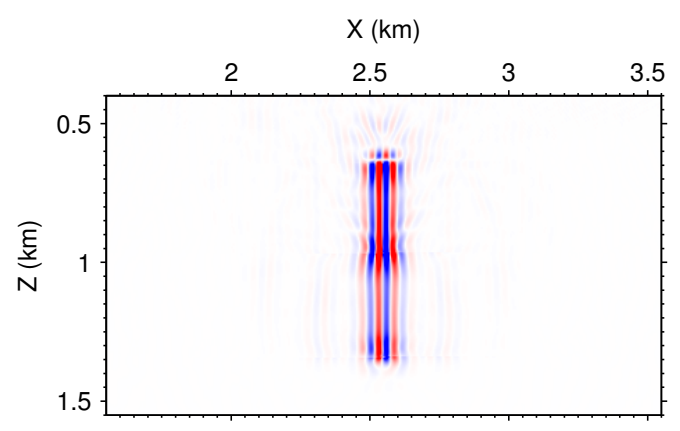

(c) $I_{\mathrm{PP}}^{1}+I_{\mathrm{PP}}^{\mathrm{r}}$

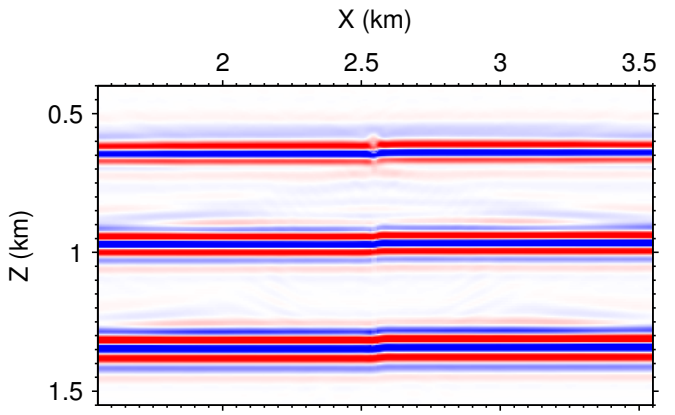

(b) $I_{\mathrm{PP}}^{\mathrm{d}}$

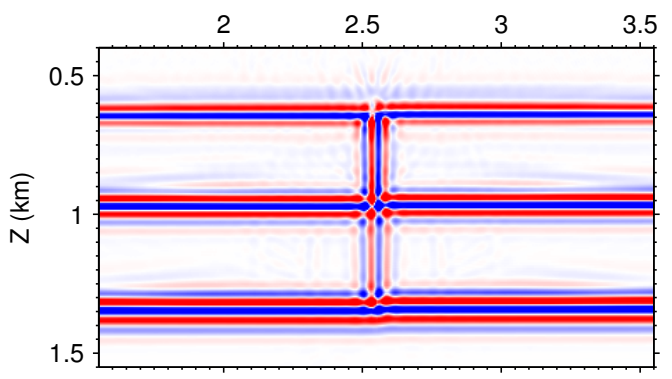

(d) Our ERTM $\left(I_{\mathrm{PP}}^{\mathrm{d}}+I_{\mathrm{PP}}^{\mathrm{l}}+I_{\mathrm{PP}}^{\mathrm{r}}\right)$

Fig. 2. PP images of a section of the fault model in Fig. 1 (a) The conventional ERTM image, (b) downward-looking image $I_{\mathrm{PP}}^{\mathrm{d}}$, (c) horizontal-looking image $\left(I_{\mathrm{PP}}^{\mathrm{l}}+I_{\mathrm{PP}}^{\mathrm{r}}\right)$, and (d) our new ERTM image $\left(I_{\mathrm{PP}}^{\mathrm{d}}+I_{\mathrm{PP}}^{1}+I_{\mathrm{PP}}^{\mathrm{r}}\right)$.

wavefield and upgoing receiver wavefield, the resulting downward-looking image (Fig. 2p) is free of background artifacts and clearly shows the horizontal reflectors. Note that the offset at the location of the fault is very small. Thus it is a challenge to infer the geometry of the fault based on the displacement of the horizontal layers, as is usually performed for conventional seismic migration methods that use only primary reflections.

If we separate the source and receiver wavefields into left-going and rightgoing components, the cross correlation between the separated right-going source 


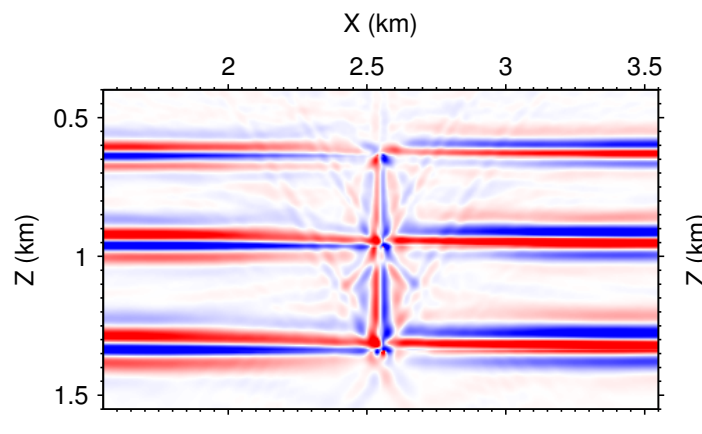

(a) Conventional ERTM

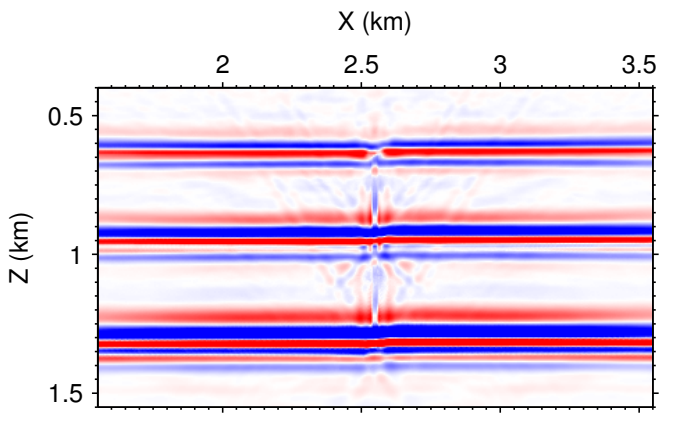

(b) With polarity correction

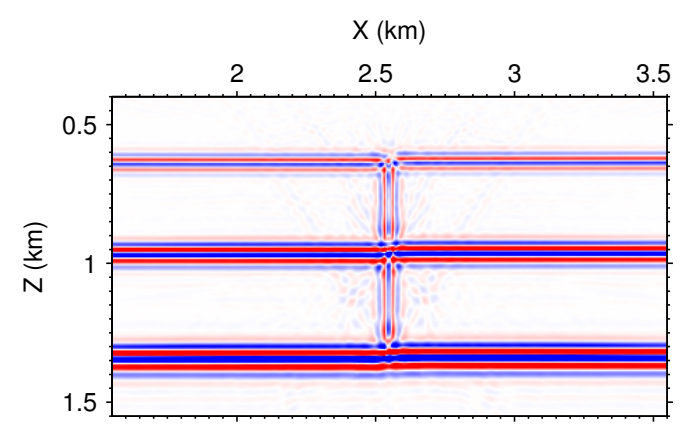

(c) Our ERTM $\left(I_{\mathrm{PS}}^{\mathrm{d}}+I_{\mathrm{PS}}^{\mathrm{l}}+I_{\mathrm{PS}}^{\mathrm{r}}\right)$

Fig. 3. PS images of a section of the fault model in Fig. 1. (a) The conventional ERTM image using Eq. (3), (b) the polarity corrected image using Eq. 6), and (c) our new ERTM image using Eq. (7).

wavefield and left-going receiver wavefield provides a right-looking view of the vertical or steeply-dipping reflectors, i.e., the fault. Similarly, the cross correlation between the left-going source wavefield and right-going receiver wavefield provides a left-looking view of the fault. The combination of the right-looking and left-looking images yields a horizontal-looking image (Fig.22), which clearly shows the fault without the background artifacts.

Since the shots are on the surface, the upward-looking image contains more artifacts than real structures. We thus combine only the downward-looking and 
horizontal-looking images to form our final image (Fig. 2d). Our new ERTM produces a greatly improved image (Fig. $2 \mathrm{~d}$ ) when compared to the conventional ERTM (Fig. 2a), clearly revealing both the horizontal layers and the vertical fault without the contamination from low-wavenumber background artifacts.

PS images of ERTM are shown in Fig. 3. There is no background artifact problem in the PS images, but the interfaces in the image obtained using the conventional imaging condition (Fig. 3a) are not coherent because of the polarity reversal. With the polarity correction using Poynting vectors for the full wavefields (Eq. (6)), the coherency of the imaged horizontal reflectors is improved (Fig. 3b), but the fault is still not well mapped. This demonstrates that, for ERTM with full wavefields, the usefulness of the polarity correction using Poynting vectors for imaging the vertical fault is much less significant than that for horizontal reflectors. Using our ERTM method that computes Poynting vectors and employs the imaging condition based on separated wavefields (Eq. (7)), we obtain a much improved image of the fault and horizontal layers (Fig. 36). Note that the resolution of the PS image (Fig. 3b) is higher than that of the PP image (Fig. 2d) because of the shorter wavelengths of $\mathrm{S}$ waves compared to $\mathrm{P}$ waves.

Because we use explosive sources in the simulation, we only show PP and PS images for illustration. If the sources generate enough $\mathrm{S}$ components using shear-wave sources, we can also produce SP and SS images in a similar approach. The SP and SS images are supposed to have features similar to the PS and PP images, respectively, except that the SS image has higher resolution than the PP image. However, if the sources contain $\mathrm{S}$ components, the wavefields become more complicated, and more artifacts may be expected in the resulting images compared to the case of explosive sources. 


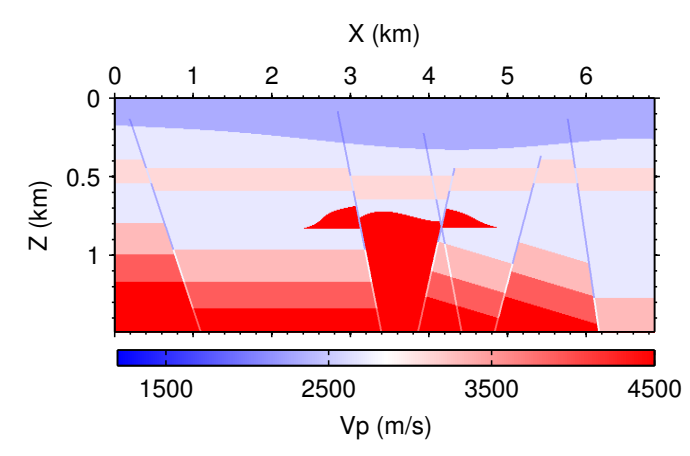
ings. (a) P-wave velocity

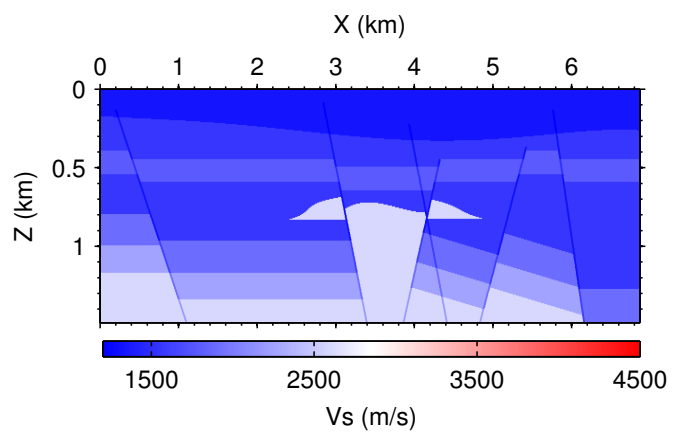

(b) S-wave velocity

Fig. 4. The velocity model for the Soda Lake geothermal site. (a) P-wave velocity and (b) S-wave velocity.

\subsection{Soda Lake geothermal model}

We next apply our ERTM method to a velocity model from the Soda Lake geothermal site to demonstrate the capability of our method for directly imaging steep faults in a complex structure environment representative of geothermal fields. The Soda Lake geothermal site is located about $10 \mathrm{~km}$ northwest of Fallon, Nevada. The velocity model is constructed by integrating well logs, gravity, seismic reflection data, and geological interpretation results. The model contains multiple stratigraphic layers and six steeply-dipping fault zones with a width of $25 \mathrm{~m}$ (Fig. 4). A high-velocity basaltic unit is intruded from the bottom to a depth of around $0.7 \mathrm{~km}$ (Magma Energy (U.S.) Corp., 2011). S-wave velocities have a structure similar to that of $\mathrm{P}$-wave velocities with a constant ratio of $V_{\mathrm{P}} / V_{\mathrm{S}}=1.73$. The velocity values of the fault zones are $15 \%$ lower than those of the surround- 


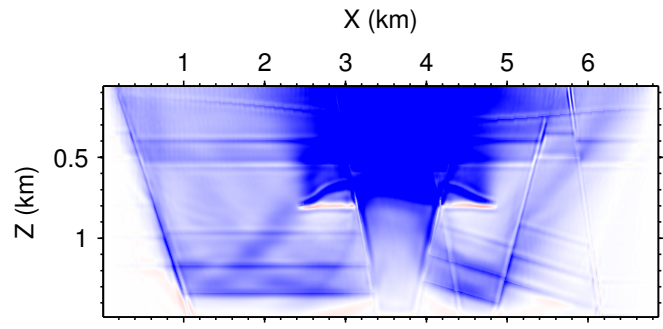

(a) Conventional ERTM

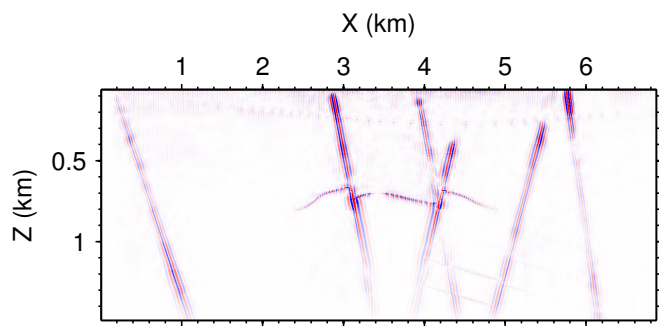

(c) $I_{\mathrm{PP}}^{1}+I_{\mathrm{PP}}^{\mathrm{r}}$

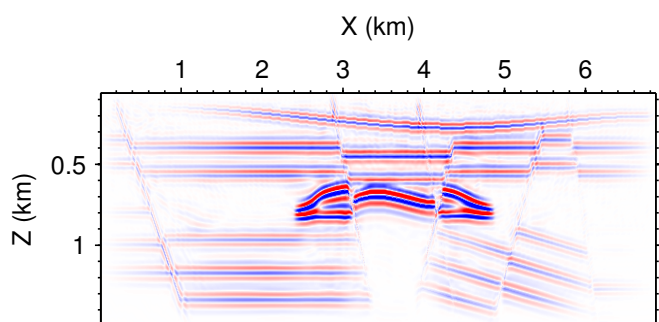

(b) $I_{\mathrm{PP}}^{\mathrm{d}}$

$\mathrm{X}(\mathrm{km})$

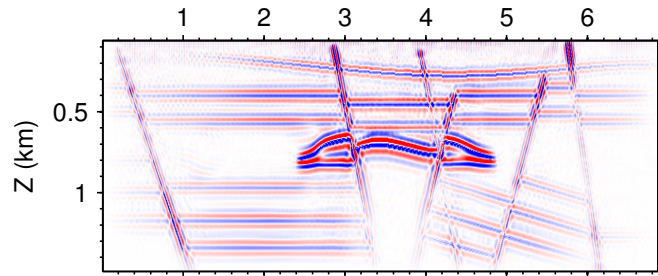

(d) Our ERTM $\left(I_{\mathrm{PP}}^{\mathrm{d}}+I_{\mathrm{PP}}^{1}+I_{\mathrm{PP}}^{\mathrm{r}}\right)$

Fig. 5. PP images of the model in Fig. 4 (a) The conventional ERTM image, (b) downwardlooking image $I_{\mathrm{PP}}^{\mathrm{d}}$, (c) horizontal-looking image $\left(I_{\mathrm{PP}}^{\mathrm{l}}+I_{\mathrm{PP}}^{\mathrm{r}}\right)$, and (d) our new ERTM image $\left(I_{\mathrm{PP}}^{\mathrm{d}}+I_{\mathrm{PP}}^{\mathrm{l}}+I_{\mathrm{PP}}^{\mathrm{r}}\right)$.

For the PP images, ERTM with the conventional imaging condition (Eq. (3)) produces high-amplitude, low-wavenumber image artifacts throughout the image, particularly above the high-contrast basaltic unit (Fig. 5a). These low-wavenumber artifacts contaminate the image so that some structures, especially the shallow ones above the high-velocity basaltic unit, are almost invisible. Using our new ERTM imaging condition with separated wavefields (Eq. (4)), the low-wavenumber artifacts are eliminated and many previously masked structures are clearly re- 
vealed (Figs. 5b-d). Horizontal or gently-dipping discontinuities are shown in the downward-looking image (Fig. 5b), while steeply-dipping faults are shown in the horizontal-looking image (Fig. 5 F). Note that not only the location but also the end points of the faults are well imaged. For example, the images clearly show that the fourth and fifth faults from the left do not reach the surface (blind faults). Locating fault terminations is important for characterizing the hydrothermal flow in a geothermal field, but they cannot be determined using the offset of imaged horizontal layers in conventional seismic migration images. The top interfaces of the basaltic unit are faintly seen in the horizontal-looking image because of numerical diffraction of the discretized model. Comparing Figs. $5 \mathrm{a}$ with $5 \mathrm{~d}$, our new ERTM method produces an improved image with clear visualization of both horizontal layers as well as the steep-dipping fault zones.

The PS images shown in Fig. 6 do not contain low-wavenumber artifacts like those in the conventional PP images. However, the mixed polarity signs of converted shear waves result in a destructive image using the conventional imaging condition (Fig. 6a). Polarity reversal correction using Poynting vectors computed with the full wavefields (Eq. (6) leads to an improved image (Fig. 6p). However, the faults and some of the deep layers are still not well imaged. Our ERTM method computes Poynting vectors and employs the imaging conditions based on the separated wavefields (Eq. (7)), and produces a much more coherent image of both the horizontal layers and steeply-dipping faults (Fig. 6c). The PS image in Fig. 6t has higher resolution than that of the PP image in Fig. 5d because of the shorter wavelengths of the $\mathrm{S}$ waves compared to $\mathrm{P}$ waves. Some interfaces that are too close to be separated in the PP image may be distinguishable in the PS image. For example, the image of the top of the basaltic unit is merged with the image of 


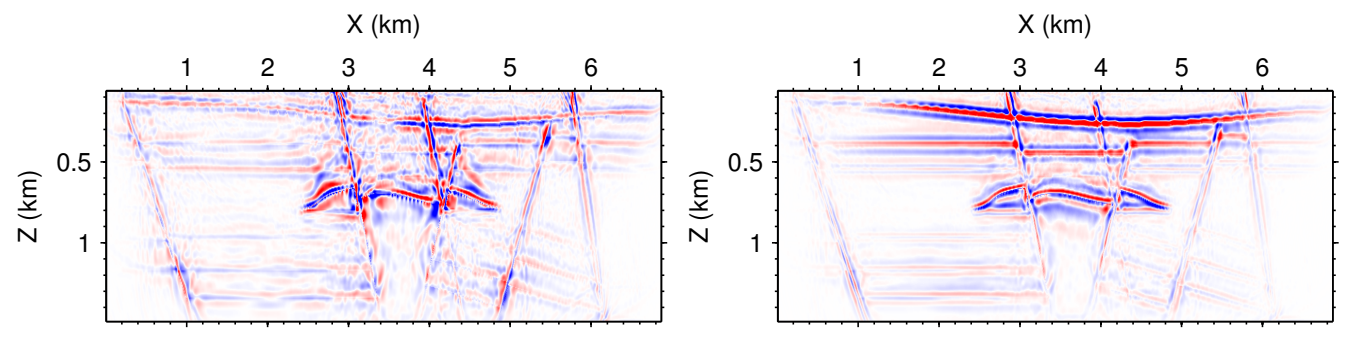

(a) Conventional ERTM

(b) With polarity correction

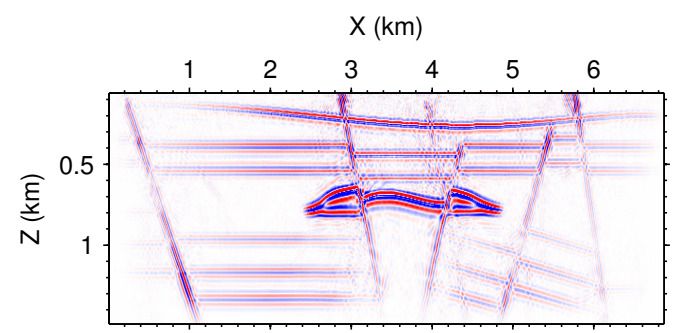

(c) Our ERTM $\left(I_{\mathrm{PS}}^{\mathrm{d}}+I_{\mathrm{PS}}^{1}+I_{\mathrm{PS}}^{\mathrm{r}}\right)$

Fig. 6. PS images of the model in Fig. 4. (a) The conventional ERTM image using Eq. 33), (b) the polarity corrected image using Eq. (6), and (c) our new ERTM image using Eq. (7).

the interface above the basaltic unit in the PP image, whereas these two interfaces are well separated in the PS image.

The computational costs for our numerical examples are approximately a couple of hours on a modern 50-CPU-core cluster.

\section{Conclusions}

We have developed a new ERTM method for directly imaging steeply-dipping fault zones in geothermal fields using multicomponent seismic data. In our new method, we first separate the forward-propagated source wavefields and the backwardpropagated receiver wavefields along the vertical direction to obtain downgoing and upgoing waves, and along the horizontal direction to obtain the left- 
going and right-going waves. We then use the separated wavefields propagating along opposite directions to produce compressional-to-compressional (or shearto-shear) images. For imaging with converted waves, we correct the polarity reversal of the shear waves using Poynting vectors computed with the separated wavefields to alleviate the multipathing issue and improve the accuracy of the estimated propagation directions. The new method has several advantages; (1) it enables us to directly image steep faults using multicomponent seismic data; (2) it eliminates the low-wavenumber background artifacts for compressional-tocompressional (or shear-to-shear) images; and (3) it is computationally efficient for polarity corrections, which is particularly important for large-scale 3D imaging. We have applied our new ERTM method to synthetic elastic seismic data generated for a Soda Lake geothermal model, and demonstrated that our method directly images steep faults as well as horizontal layers. For imaging with converted waves, our new method produces much clearer images of steeply-dipping fault zones and horizontal layers compared to those obtained using Poynting vectors and an imaging condition based on unseparated wavefields.

In our numerical examples, we use noise-free synthetic seismic data and true velocity models to perform migration and demonstrate the effectiveness of our new ERTM imaging condition. For practical application, the velocity models used for ERTM can be obtained using velocity analysis and high-resolution seismicwaveform inversion. Velocity uncertainties and noise in seismic data could degrade migration images. Nevertheless, our method can still produce better imaging results than the conventional ERTM. 


\section{Acknowledgements}

This work was supported by the Geothermal Technologies Program of the U.S. Department of Energy through contract DE-AC52-06NA25396 to Los Alamos National Laboratory. The computation was performed on super-computers provided by the Institutional Computing Program of Los Alamos National Laboratory. We thank James Echols for his help in building the velocity model for the Soda Lake geothermal site. The model is built in reference to a time migration result provided by Magma Energy (U.S.) Corp. We thank Kenneth Hanson of Los Alamos National Laboratory for his help in refining this manuscript, Associate Editor Joseph Moore and two anonymous reviewers for their valuable comments.

\section{References}

Barnicoat, A., Sheldon, H., Ord, A., 2009. Faulting and fluid flow in porous rocks and sediments: implications for mineralisation and other processes. Mineralium Deposita 44, 705-718.

Baysal, E., Kosloff, D., Sherwood, J., 1983. Reverse time migration. Geophysics 48, 1514-1524. URL: http://library.seg.org/doi/abs/10.1190/1. 1441434, doi:10.1190/1.1441434.

Baysal, E., Kosloff, D.D., Sherwood, J., 1984. A two-way nonreflecting wave equation. Geophysics 49, 132-141.

Caine, J.S., Forster, C.B., 1999. Fault zone architecture and fluid flow: Insights from field data and numerical modeling. Geophysical Monograph Series 113, $101-127$. 
Chang, W.F., McMechan, G.A., 1987. Elastic reverse-time migration. Geophysics $52,1365-1375$.

Claerbout, J.F., 1985. Imaging the Earth's Interior. Blackwell Scientific Publications, Oxford.

Corbel, S., Schilling, O., Horowitz, F., Reid, L., Sheldon, H., Timms, N., Wilkes, P., 2012. Identification and geothermal influence of faults in the Perth metropolitan area, Australia, in: Thirty-Seventh Workshop on Geothermal Reservoir Engineering, Stanford, CA.

Costa, J., Silva Neto, F., Alcântara, M., Schleicher, J., Novais, A., 2009. Obliquity-correction imaging condition for reverse time migration. Geophysics 74, S57-S66. URL: http://library. seg.org/doi/abs/10.1190/1.3110589, doi:10.1190/1.3110589, arXiv:http://library.seg.org/doi/pdf/10.1190/1.3110589.

Dellinger, J., Etgen, J., 1990. Wavefield separation in twodimensional anisotropic media. Geophysics 55, 914-919. URL: http://dx.doi.org/10.1190/1.1442906, doi:10.1190/1.1442906, arXiv:http://dx.doi.org/10.1190/1.1442906.

Denli, H., Huang, L., 2008. Elastic-wave reverse-time migration with a wavefieldseparation imaging condition. 78th Annual International Meeting, SEG, Expanded Abstracts , 2346-2350.

Dickens, T., Winbow, G., 2011. RTM angle gathers using Poynting vectors. 81st Annual International Meeting, SEG, Expanded Abstracts , 3109-3113URL: http://library.seg. 

arXiv:http://library.seg.org/doi/pdf/10.1190/1.3627841.

Du, Q., Zhu, Y., Ba, J., 2012. Polarity reversal correction for elastic reverse time migration. Geophysics 77, S31-S41.

Fomel, S., 2011. Theory of 3-D angle gathers in wave-equation seismic imaging. Journal of Petroleum Exploration and Production Technologies 1, 11-16.

Gaiser, J., Moldoveanu, N., Macbeth, C., Michelena, R., Spitz, S., 2001. Multicomponent technology: the players, problems, applications, and trends: Summary of the workshop sessions. The Leading Edge 20, 974-977.

Goyal, K., Kassoy, D., 1980. Fault zone controlled charging of a liquid-dominated geothermal reservoir. J. Geophys. Res. 85, 1867-1875.

Guitton, A., Kaelin, B., Biondi, B., 2007. Least-squares attenuation of reversetime-migration artifacts. Geophysics 72, S19-S23. URL: http://library. seg.org/doi/abs/10.1190/1.2399367, doi:10.1190/1.2399367, arXiv:http://library.seg.org/doi/pdf/10.1190/1.2399367.

Hatherton, T., Macdonald, W.J.P., Thompson, G.E.K., 1966. Geophysical methods in geothermal prospecting in new zealand. Bulletin volcanologique 29, 485-497.

Huang, L., Albrecht, M., 2011. Seismic and magneto-telluric imaging for geothermal exploration at Jemez pueblo in New Mexico. Proceedings of the ThirtySixth Workshop on Geothermal Reservoir Engineering, Stanford University , 944-949. 
Huang, L., Kelley, S., Zhang, Z., Rehfeldt, K., Albrecht, M., Kaufman, G., 2011. Imaging faults with reverse-time migration for geothermal exploration at Jemez Pueblo in New Mexico. Geothermal Resources Council Transactions 35, 833-837. URL: https://www.geothermal-library.org/index.php? mode=pubs\&action=view\&record $=1029338$

Huenges, E. (Ed.), 2010. Geothermal energy systems: exploration, development, and utilization. Wiley-VCH Verlag GmbH \& Co. KGaA. doi:10.1002/ 9783527630479 .

Kaelin, B., Guitton, A., Ortigosa, F., 2007. Illumination effects in reverse time migration. EAGE 69th Conference .

Knipe, R., 1992. Structural and tectonic modelling and its application to petroleum geology. Elsevier. volume 1 of Norwegian Petroleum Society Special Publication. chapter Faulting processes and fault seal. pp. 325-342.

Liu, F., Zhang, G., Morton, S., Leveille, J., 2011. An effective imaging condition for reverse-time migration using wavefield decomposition. Geophysics 76, S29-S39.

Loewenthal, D., Stoffa, P.L., Faria, E.L., 1987. Suppressing the unwanted reflections of the full wave equation. Geophysics 52, 1007-1012.

López, D.L., Smith, L., 1995. Fluid flow in fault zones: analysis of the interplay of convective circulation and topographically driven groundwater flow. Water Resources Research 31, 1489-1503.

Lu, R., Traynin, P., Anderson, J., 2009. Comparison of elastic and acoustic 
reverse-time migration on the synthetic elastic Marmousi-II OBC dataset. 79th Annual International Meeting, SEG, Expanded Abstracts .

Magma Energy (U.S.) Corp., 2011. Soda Lake DOE Phase I report — Soda Lake 3D-3C reflection seismic survey. Technical Report. Magma Energy (U.S.) Corp.

McMechan, G.A., 1983. Migration by extrapolation of time-dependent 口 boundary values. Geophysical Prospecting 31, 413-420. URL: http: //dx.doi.org/10.1111/j.1365-2478.1983.tb01060.x, doi:10.1111/j. 1365-2478.1983.tb01060.x.

Megies, T., Wassermann, J., 2014. Microseismicity observed at a nonpressure-stimulated geothermal power plant. Geothermics 52, 36 49. URL: http://wWw.sciencedirect.com/science/article/pii/ S0375650514000030, doi:10.1016/j.geothermics.2014.01.002

Mulder, W.A., Plessix, R.E., 2004. A comparison between one-way and two-way wave-equation migration. Geophysics 69, 1491-1504.

Patrikeeva, N., Sava, P., 2013. Comparison of angle decomposition methods for wave-equation migration. 83rd Annual International Meeting, SEG, Expanded Abstracts , 3773-3778URL: http://library.seg.org/ doi/abs/10.1190/segam2013-0780.1, doi:10.1190/segam2013-0780.1. arXiv:http://library.seg.org/doi/pdf/10.1190/segam2013-0780.1.

Rickett, J.E., Sava, P.C., 2002. Offset and angle-domain common image-point gathers for shot-profile migration. Geophysics 67, 883-889. 
Sava, P., Fomel, S., 2006. Time-shift imaging condition in seismic migration. Geophysics 71, S209-S217.

Stewart, R., Gaiser, J., Brown, R., Lawton, D., 2002. Convertedwave seismic exploration: Methods. Geophysics 67, 1348-1363. URL: http://dx .doi .org/10.1190/1.1512781, doi:10.1190/1.1512781, arXiv:http://dx.doi.org/10.1190/1.1512781

Stewart, R., Gaiser, J., Brown, R., Lawton, D., 2003. Convertedwave seismic exploration: Applications. Geophysics 68, 40-57. URL: http://dx.doi .org/10.1190/1.1543193, doi:10.1190/1.1543193, arXiv:http://dx.doi.org/10.1190/1.1543193

Tan, S., Huang, L., 2014. Least-squares reverse-time migration with a wavefieldseparation imaging condition and updated source wavefields. Geophysics 79, S195-S205.

Vyas, M., Nichols, D., Mobley, E., 2011. Efficient RTM angle gathers using source directions. 81st Annual International Meeting, SEG, Expanded Abstracts .

Wassing, B., van Wees, J., Fokker, P., 2014. Coupled continuum modeling of fracture reactivation and induced seismicity during enhanced geothermal operations. Geothermics 52, 153-164. URL: http://www. sciencedirect.com/science/article/pii/S0375650514000558, doi:10.1016/j.geothermics.2014.05.001.

Whitmore, N., 1983. Iterative depth migration by backward time propagation. 53rd Annual International Meeting, SEG, Expanded Abstracts . 
501

Yan, J., Sava, P., 2008. Isotropic angle-domain elastic reverse-time migration. Geophysics 73, 229-239. doi:10.1190/1.2981241.

Yoon, K., Guo, M., Cai, J., Wang, B., 2011. 3D RTM angle gathers from source wave propagation direction and dip of reflector. 81st Annual International Meeting, SEG, Expanded Abstracts .

Yoon, K., Marfurt, K., Starr, E.W., 2004. Challenges in reverse-time migration. 74th Annual International Meeting, SEG, Expanded Abstract , 10571060doi:10.1190/1.1851068.

Youn, O.K., Zhou, H., 2001. Depth imaging with multiples. Geophysics 66, 246-255. URL: http://library.seg. org/doi/abs/10.1190/1.1444901, doi:10.1190/1.1444901, arXiv:http://library.seg.org/doi/pdf/10.1190/1.1444901. 